\section{Bioactive Molecules: Translating Chemical and Biological Information from Yeast through Arabidopsis to Crops}

\section{Cecilia Rodriguez-Furlán, Patricio Pérez-Henríquez and Lorena Norambuena*}

${ }^{1}$ Plant Molecular Biology Centre, Department of Biology, Faculty of Sciences, Universidad de Chile, Las Palmeras 3425 Nunoam, Santiago, Chile

*Corresponding author: Lorena Norambuena, Plant Molecular Biology Centre, Department of Biology, Faculty of Sciences, Universidad de Chile, Las Palmeras 3425 Nunoa, Santiago, Chile, Tel: (2) 29787193; E-mail: Inorambuena@u.uchile.cl, norambuena.l@gmail.com

Rec date: May 29, 2015; Acc Date: June 04, 2015; Pub date: June 06, 2015

Copyright: (c) 2015 Rodriguez-Furlán C, et al. This is an open-access article distributed under the terms of the Creative Commons Attribution License, which permits unrestricted use, distribution, and reproduction in any medium, provided the original author and source are credited.

\section{Letter to the Editor}

Chemical tools have been extensible used to probe complex biological processes [1]. A variety of small molecules $(<500 \mathrm{Da})$ like Brefeldin A, Tyrphostin A23, Wortmannin have been intensively utilized to study endomembrane protein trafficking and have illustrated corresponding biological functions [2-7]. After the application, bioactive small molecules can be rapidly permeated or transported into cells to create observable effects. These molecules can be washed out to reverse their effects, permitting a return to a normal state. This rapid and reversible behavior along with precise concentration and time of treatment provides a high degree of control, permitting dynamic processes to be studied in vivo.

In the past decade several academic and company research initiatives undertook the systematic design and synthesis of small molecules $(<500 \mathrm{Da})$ and their subsequent use as probes for different biological processes in diverse organisms. As a result several collections of bioactive compounds, chemical libraries, became available for the research community. These libraries consist of lowmolecular weight compounds synthesized by combinatorial chemistry with defined properties according to the Lipinski rule of five $[8,9]$. These rules outlines favorable physicochemical properties to get bioactive compounds, molecular weight $(\mathrm{Da}) \leq 500, \log \mathrm{P}$ (octanol/ water partition coefficient) $\leq 5$, Number of $\mathrm{H}$-bond donor $\leq 5$, Number of $\mathrm{H}$-bond acceptor $\leq 10$, Rotatable bonds $\leq 12$ [8]. Application of small bioactive molecules as a strategy to systematically screen for novel modifiers of a biological phenomenon of interest have gained increasing attention [10]. These approach, named chemical genomics, combines chemistry and biology along with bioinformatics which is required for data mining, structure analysis, data sharing and the extraction of useful data [11]. In principle, a chemical genomic screen can be performed in any system.

A chemical genomics high throughput screening (HTS) in Saccharomyces cerevisiae allowed to identify compounds that interfere with the delivery of the vacuolar resident protein carboxypeptidase $\mathrm{Y}$ (CPY) [12]. Among those compounds was selected Sortin2. Structureactivity relationship (SAR) studies identified active and inactive homologs of this compound (Norambuena et al.,). Sortin2 contains a chlorobenzene, a furan, a thiazolidine ring, and a sulphite group. The sulphite group was essential to the activity of the compound. The data suggest that the interaction between Sortin 2 and its target most likely requires a dense electron cloud to affect the target activity and function [13].
A

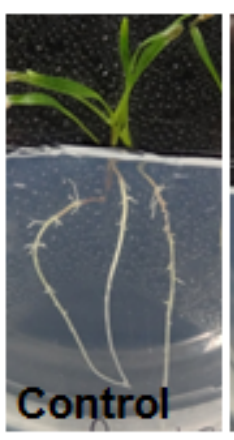

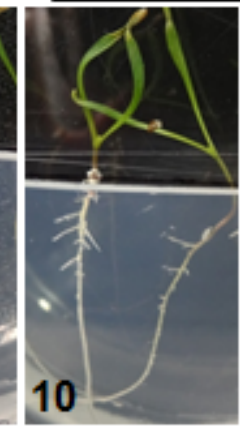
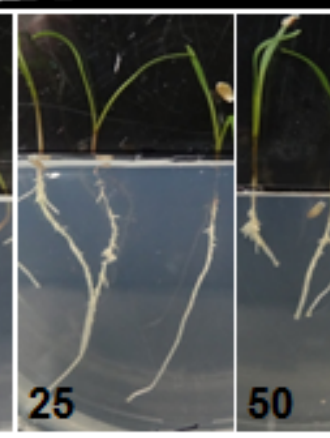

Sortin2

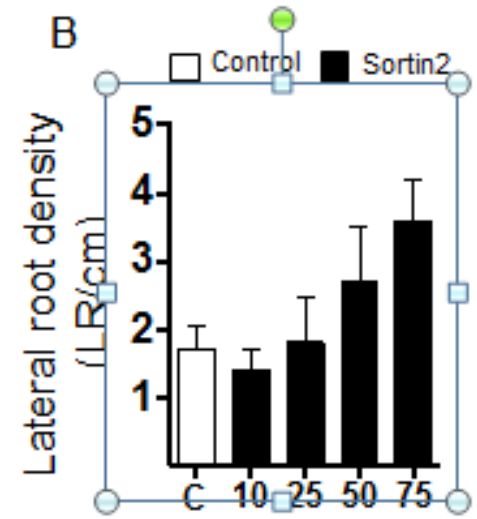

Figure 1: A compound identified by chemical genomics modifies root system architecture in carrots. 7 day-old carrot seedlings were transferred to Control and different concentrations of Sortin2 (10 to $75 \mu \mathrm{g} / \mathrm{mL})$. A. Whole plant images of carrot seedlings after seven days of Sortin 2 treatment. B. Lateral root density quantification of control and Sortin 2 treated plants. mean + SEM. N=6.

Sorting2, was also capable to interfere with the delivery of the vacuolar of CPY in the model plant Arabidopsis [12]. Interestingly in this plant Sortin2 modifies the root architecture increasing lateral root occurrence and inhibiting principal root growth increasing overall lateral root density (LRD) [14]. An increase in LRD is a desirable trait in crops as it optimizes water and nutrient uptake. Thus, translating all 
Citation: Rodriguez-Furlán C, Pérez-Henríquez P, Norambuena L (2015) Bioactive Molecules: Translating Chemical and Biological Information from Yeast through Arabidopsis to Crops. Biochem Anal Biochem 4: 186. doi:10.4172/2161-1009.1000186

Page 2 of 2

the chemical and biological information available for Sortin 2 could be effective to improve crop yields. We examined Sortin2 effect in carrot (Daucus carota). Carrots are characterized by a strong, deep, welldeveloped root system. Lateral root development throughout is very poor [15]. A dry surface soil tends to promote a vigorous development of strong laterals roots from the deeper portions of the root. Thus, generating more lateral root would be a desirable trait to overcome stress. We tested the modification of carrot roots by means of chemical stimulation with Sortin2. Roots of seven day-old seedlings were exposed to Sortin 2 for additional seven days. Aerial organs of Sortin2treated plants did not showed color or turgor differences from control plants (Figure 1A). Meanwhile, root was strongly modified (Figure $1 \mathrm{~A})$. Figure $1 \mathrm{~B}$ shows that the lateral root density was increased in a dose-dependent manner in Sortin2 treated plants. The highest Sortin2 concentration tested, $75 \mu \mathrm{g} / \mathrm{mL}$, trigger a two-fold increase in lateral root density.

These results indicates that chemical information and action can be translated from yeast through model plants as Arabidopsis and ultimately to crops. We emphasizes on the critical role that chemistry is playing in unraveling physiological processes in plants, and how these new insights allow to suggest novel approaches to improve yield in crops.

\section{Acknowledgement}

This work has been supported by FONDEF CA12I10206, FONDECYT 1120289, ANILLO ACT 1110, VIU 110058.

\section{References}

1. Wijdeven RH, Neefjes J, Ovaa H (2014) How chemistry supports cell biology: the chemical toolbox at your service. Trends Cell Biol 24: 751-760.

2. Dambournet D, Machicoane M, Chesneau L, Sachse M, Rocancourt M, et al. (2011) Rab35 GTPase and OCRL phosphatase remodel lipids and Factin for successful cytokinesis. Nat Cell Biol 13: 981-988.

3. Geldner N, Anders N, Wolters H, Keicher J, Kornberger W, et al. (2003) The Arabidopsis GNOM ARF-GEF mediates endosomal recycling, auxin transport, and auxin-dependent plant growth. Cell 112: 219-230.
4. Kleine-Vehn J, Dhonukshe P, Sauer M, Brewer PB, WiÅıniewska J, et al. (2008) ARF GEF-dependent transcytosis and polar delivery of PIN auxin carriers in Arabidopsis. Curr Biol 18: 526-531.

5. Nebenführ A, Ritzenthaler C, Robinson DG (2002) Brefeldin A deciphering an enigmatic inhibitor of secretion. Plant Physiol 130: 1102-1108.

6. Reichardt I, Stierhof YD, Mayer U, Richter S, Schwarz H, et al. (2007) Plant cytokinesis requires de novo secretory trafficking but not endocytosis. Curr Biol 17: 2047-2053.

7. TakÃ Ä̈ T, Pechan T, SamajovÃ $;$ O, OveÄka M, Richter H, et al. (2012) Wortmannin treatment induces changes in Arabidopsis root proteome and post-Golgi compartments. J Proteome Res 11: 3127-3142.

8. Lipinski CA, Lombardo F, Dominy BW, Feeney PJ (2001) Experimental and computational approaches to estimate solubility and permeability in drug discovery and development settings. Adv Drug Deliv Rev 46: 3-26.

9. Shelat AA, Guy RK (2007) Scaffold composition and biological relevance of screening libraries. Nat Chem Biol 3: 442-446.

10. Lamberth C, Jeanmart S, Luksch T, Plant A (2013) Current challenges and trends in the discovery of agrochemicals. Science 341: 742-746.

11. Robert S, Raikhel NV, Hicks GR (2009) Powerful partners: Arabidopsis and chemical genomics. Arabidopsis Book 7: e0109.

12. Zouhar J, Hicks GR, Raikhel NV (2004) Sorting inhibitors (Sortins): Chemical compounds to study vacuolar sorting in Arabidopsis. Proc Natl Acad Sci U S A 101: 9497-9501.

13. Norambuena L, Zouhar J, Hicks G, Raikhel, N (2008) Identification of cellular pathways affected by Sortin, a synthetic compound that affects protein targeting to the vacuole in Saccharomyces cerevisiae. BMC Chemical Biology 8: 1.

14. Perez-Henriquez P, Raikhel NV, Norambuena L (2012) Endocytic trafficking towards the vacuole plays a key role in the auxin receptor SCF(TIR)-independent mechanism of lateral root formation in A. thaliana. Molecular plant 5: 1195-1209.

15. Thorup-Kristensen K, van den Boogaard R (1999) Vertical and horizontal development of the root system of carrots following green manure. Plant and Soil 212: 143-151. 